\title{
3 Research Square

\section{Transcriptome Profiling Reveals Key Genes In Regulation Of The Tepal Trichome Development In Lilium Pumilum D.C.}

\section{Yin Xin}

China Agricultural University

\section{Wenqiang Pan}

China Agricultural University

\section{Xi Chen}

Beijing Academy of Agriculture and Forestry Sciences

Yixin Liu

Beijing Academy of Agriculture and Forestry Sciences

\section{Mingfang Zhang}

Beijing Academy of Agriculture and Forestry Sciences

\section{Xuqing Chen}

Beijing Academy of Agriculture and Forestry Sciences

\section{Fengping Yang}

Beijing Academy of Agriculture and Forestry Sciences

Jingru Li

China Agricultural University

Jian Wu

China Agricultural University

Yunpeng Du ( $\nabla$ dyp_851212@126.com )

Beijing Academy of Agriculture and Forestry Sciences https://orcid.org/0000-0001-7188-1309

\section{Xiuhai Zhang}

Beijing Academy of Agriculture and Forestry Sciences

\section{Research Article}

Keywords: Lily, Lilium pumilum, tepal, trichome, transcriptome

Posted Date: April 27th, 2021

DOl: https://doi.org/10.21203/rs.3.rs-424931/v1 
License: (c) (i) This work is licensed under a Creative Commons Attribution 4.0 International License. Read Full License

Version of Record: A version of this preprint was published at Plant Cell Reports on July 14th, 2021. See the published version at https://doi.org/10.1007/s00299-021-02753-x. 


\section{Abstract}

Trichome is a specialized structure found on the surface of the plant with important function in survival against abiotic and biotic stress. It is also an important economic trait in crop breeding. Extensive research has investigated the foliar trichome in model plants (Arabidopsis and tomato). However, the developmental mechanism of tepal trichome remains elusive. Lilium pumilum is an edible ornamental bulb and a good breeding parent possessing cold and salt-alkali resistance. Here, we found a natural mutant of Lilium pumilum grown on a highland whose tepals are covered by trichomes. Our data indicate that trichomes of this mutant are multicellular and branchless. Notably, stomata are also developed on the tepal of the mutant as well, suggesting there may be a correlated between trichome and stomata regulation. Furthermore, we isolated 27 differentially expressed genes (DEGs) by comparing the transcriptome profiling between the natural mutant and the wild type. These twenty-seven genes belong to four groups: epidermal cell cycle and division, trichome morphogenesis, stress response, and transcription factors. Quantitative real-time PCR in Lilium pumilum (natural mutant and the wild type) and other lily species (Lilium leichtlinii var. maximowiczii/ trichome; Lilium davidii var. willmottiael, trichomeless) confirmed the validation of RNA-seq data and identified several trichome-related genes.

\section{Key Message}

A number of potential genes and pathways involved in tepal trichome development were identified in a natural lily mutant by transcriptome analysis and were confirmed with trichome and trichome-less species.

\section{Introduction}

Plant have specialized structures that are differentiated from epidermal cells, and grow on the plant epidermis, called trichomes (Hulskamp 2004). Trichomes are generally composed of head cells, stalk cells, and basal cells, and are mainly divided into three types: single-cellular or multi-cellular types, glandular or glandless types, and branched or branchless types. The morphogenesis and temporal-spatial distribution of trichomes are strictly regulated by conditions such as signal transductions and hormones, which make trichomes fine structures for studying cell differentiation, cell fate, and morphological mechanism (Yang and Ye 2013). Trichomes serve as a natural barrier between the plant surface and its environment, and thus play a crucial role in protecting against biotic and abiotic stress, such as protecting plants from pathogens, preventing ultraviolet (UV) radiation damage, and providing drought resistance (Bac-Molenaar et al. 2019; Tang et al. 2020; Ozturk Cali and Karavin 2020). Apart from protecting the plant as a barrier, trichomes also have a great impact on human life. The glandular trichomes of Artemisia annua can produce artemisinin which is a known vital antimalarial drug (Zhou et al. 2020). Furthermore, cotton trichomes are an important resource for fiber and textile production, with enormous economic value (Ma et al. 2016). Therefore, trichomes are economically important for crop breeding. 
The morphogenesis and molecular regulation mechanism of trichomes in Arabidopsis have been well studied. Trichomes in Arabidopsis are single-celled and branched. After four endoreduplications in the rosette leaf of Arabidopsis, incipient trichomes rapidly expand out of the leaf surface and constantly stretch outwards to form rod-like structures. The trichomes also initiate branches and the top tip of the stalk, eventually becoming sharp (Yanagisawa et al. 2015; Seale et al. 2018; Okamoto et al. 2020). All the above processes have strict regulatory mechanisms and depend on the precise gene expression, especially some essential transcription factors (TFs) (Wang et al. 2019). TFs including the R2R3 MYB protein (GLABRA1/ GL1), the WD40-repeat protein (TRANSPARENT TESTA GLABRA1/ TTG1), and the basic helix-loop-helix TFs (GLABRA 3/ GL3 and ENHANCER OF GLABRA3/ EGL3) form a crucial activator complex to activate GLABRA2 (GL2) expression. GL2 is an HD-ZIP IV domain transcription factor, which directly promotes trichome initiation (Morohashi et al. 2007; Bloomer et al. 2012; Khosla et al. 2014; Airoldi et al. 2019). Furthermore, TTG2, a member of WRKY family, acts downstream of TTG1 and GL1. TTG2 has a redundant effect with GL2 in regulating trichome outgrowth (Li et al. 2015a). MYB family transcription factors TRIPTYCHON and CAPRICE (CPC) compete with GL1 to combine with GL3 and EGL3, and negatively regulate trichome development (Doroshkov et al. 2019). Similar to TRY and CPC, ENHANCER OF TRY AND CPC1 (ETC1) also inhibits trichome occurrence (Kirik et al. 2004).

Overexpression of TRICHOMELESS 1 inhibits the function of GL1 and the CIN-TCP family transcription factor TCP4, and suppresses trichome initiation by directly activating TCL1 (Zhang et al. 2018; Vadde et al. 2019).

In most plants, such as cucumber, tomato, tobacco, trichomes are multi-cellular. On cucumber fruits, the trichome is also called a 'fruit thorn' and has two types: glandular and non-glandular types. The glandular type has a head-like structure, while the non-glandular type has a spherical base and a long rod-like structure (Chen et al. 2014). In rice, trichomes are grouped into long hair, micro hair, and glandular hair types. Long hair is mainly distributed on the silicon cells of the vascular bundles, while micro hair and glandular hair are distributed around the stomata or motor cells (Wang et al. 2013). In fact, the molecular mechanism of trichome development is somewhat variable. For example, AtCPC negatively regulates trichome development in Arabidopsis but makes no difference in tomatoes (Tominaga-Wada et al. 2013). The R3 MYB transcription factor gene Oryza sativa TRICHOMELESS1 (OsTCL1) significantly inhibits trichome formation in Arabidopsis but has little effect on trichome formation in transgenic rice (Zheng et al. 2016).

Lilium pumilum is a perennial herbaceous plant in monocots that is native to China, and is used as a garden plant, potted flower, and a breeding resource. In this study, we found a natural mutant in the Lilium pumilum distribution area, of which flower buds are covered by trichomes. Previous studies have shown that the presence of tepal trichome provides resistance to various types of stress. For example, trichomes in tomatoes provide enhanced resistance to western flower thrips (Escobar-Bravo et al. 2017). Glandular trichomes on the flower are able to secrete chemicals to prevent herbivores feeding in the wild (Livingston et al. 2020). 
In this study, we found that the trichome on Lilium pumilum belongs to the multicellular and branchless type, as observed by electron microscopy. We compared the transcriptome profiling between the wild type (trichomeless) and the natural mutant (trichome) of Lilium pumilum. Our data indicate that DEGs related to trichome development are mainly enriched on the aspects of cell structure formation, cell division, and material metabolism. Finally, we identified several possible candidate genes in trichome development regulation, which are related to transcription factors, stress responsiveness, trichome morphogenesis, and epidermal cell cycle. Quantitative real-time PCR in Lilium pumilum (the mutant and wild type) and lily species (Lilium leichtlinii var. maximowiczii, trichome; Lilium davidii var. willmottiae, trichomeless) confirmed the differential expression of several DEGs and verified several trichome-related genes. This study provides some clues to trichome development in lily and contributes to lily's resistant breeding.

\section{Materials And Methods}

\section{Plant materials}

Lilium pumilum plants were grown in National germplasm resources of lily at Beijing Academy of Agriculture and Forestry Sciences. Tepals of trichome/ trichomeless flower buds $(13.5 \mathrm{~mm}$ and $33.5 \mathrm{~mm}$ in length) were taken for assays. In addition, two Asiatic cultivars, Lilium leichtlinii var. maximowiczii (trichome) and Lilium davidii var. willmottiae (trichomeless), were also used for confirmation by quantitative real-time PCR. The flower buds were $10 \mathrm{~mm}$ and $30 \mathrm{~mm}$ in length.

Transmission electron microscope (TEM) analysis \& Scanning electron microscope (SEM) analysis

Tepals of Lilium pumilum were divided into small sections. For SEM analysis, the samples were fixed with $3 \%(\mathrm{w} / \mathrm{v})$ glutaraldehyde for 3 hours (h), and then washed with normal saline for 3 times, soaked for 10 minutes ( $\mathrm{min}$ ), and finally washed with normal saline for 3 times. Gradient dehydration was performed with different concentrations of ethanol $(80 \%, 90 \%, 95 \%, 100 \%$ ethanol; v/v) for $10 \mathrm{~min}$, and then dried naturally. The dried samples were observed under SEM after being sprayed with gold-palladium. For TEM analysis, the samples were first fixed in $2.5 \%(\mathrm{v} / \mathrm{v})$ glutaraldehyde. After rinsed with PBS buffer $(\mathrm{pH} 7)$ and fully dehydrated with acetone, the samples were embedded and then were sliced by an ultrathin microtome. Finally, the ultrathin sections were dyed with uranium acetate staining solution and then observed by TEM.

RNA extraction and quantitative real-time PCR analysis

By following the instruction manual, total RNA was extracted from different samples using RNAprep Pure Plant Plus Kit (Tiangen). Then, the first-strand CDNA was synthesized according to the manual of PrimeScript RT reagent Kit with gDNA Eraser (TaKaRa). Quantitative real-time PCR (qRT-PCR) analysis was used to verify the expression levels of selected genes from RNA-seq data. The specific primers for qRT-PCR were generated by GenScript tool (https://www.genscript.com/tools/real-time-pcr-taqmanprimer-design-tool\#) and the of primer sequences are listed in Table S1. 18S rRNA was used as the internal reference gene (Wu et al. 2019; Li et al. 2015b). All qRT-PCR assays were performed with at least 
three biological replicates. The qRT-PCR experiment was conducted by using TB Green ${ }^{\circledR}$ Premix Ex Taq ${ }^{\text {TM }}$ II (TaKaRa) in ABI 7500 Fast Real-time PCR system.

RNA-seq data processing and de novo assembly

After total RNA was qualified, mRNA was enriched with Oligo magnetic beads and then fragmented to create CDNA libraries by adding fragmentation buffer. Agilent 2100 was used to detect the insert size of the library for ensuring the quality of libraries (Nachamkin et al. 2001). All libraries were sequenced with the Hiseq X system (Illumina) by Novogene (Beijing). The sequenced reads (raw reads) were filtered by removing low-quality reads (Data will be submitted to NCBI SRA once the manuscript is accepted).

Finally, clean reads were obtained. Trinity software was used to assemble the clear reads (Grabherr et al. 2011). After clustering de novo assembled transcripts and counting overlapping reads, unigenes were obtained for further analysis.

Gene annotation, enrichment, and differential expression analysis

The unigenes were blasted against GenBank Non-redundant, Protein family (Pfam), Swiss-Prot, Karyotic Ortholog Groups (KOG), Gene Ontology databases (GO) respectively to obtain the annotation information. Differential expression analysis of genes was further conducted by analyzing GO and KEGG (Kyoto Encyclopedia of Genes and Genomes) functional enrichment (Kanehisa et al. 2008). The method for GO enrichment analysis was GOseq software, based on Wallenius non-central Hyper distribution (Young et al., 2010). In addition, the KOBAS (2.0) software was used to identify KEGG pathways (Mao et al. 2005). The FDR of the enrichment pathway was less than 0.05 or above 2 .

The 'eFP-Seq' Brower analysis

By comparing transcriptome specific unigenes with NCBI database (https://blast.ncbi.nlm.nih.gov/Blast.cgi), the homologous genes in Arabidopsis thaliana were obtained. The Arabidopsis 'eFP-Seq Browser' tool can present the relative expression level of genes through 'electronic fluorescent pictographic' (eFP) images, which is used to observe the differential expression of genes (http://bar.utoronto.ca/efp/cgi-bin/efpWeb.cgi) (Sullivan et al. 2019). In this study, 'Tissue-specific' part including trichome was analyzed.

\section{Statistical analysis}

Three biological replicates were analyzed for each condition. The GraphPad Prism (version 7) software was used for Student's $t$-test verification (Mitteer et al. 2020). Significant differences were marked as * $p<$ 0.05 and ${ }^{* *} p<0.01$.

\section{Results}

Morphological and histological analysis of trichome 
The tepals of Lilium pumilum with trichome (natural mutant; TR) and trichomeless (wild type; TL) at 13.5 $\mathrm{mm}$ (green tepal) and $33.5 \mathrm{~mm}$ (tepal coloring) in length, respectively, were used in this study (Fig. 1a, b). The SEM assay showed that the epidermis of the mutant has a large number of hairs, while relatively flat on TL's epidermis (Fig. 1c, d). Moreover, we found that the trichomes of Lilium pumilum were of the multicellular and branchless type (Fig. 1c). To further characterize the trichome, we carried out the TEM observation. In the cross section of the trichome, we could see the cell wall, vacuole, and other organelles (Fig. 1e). We also observed that the trichome was composed of multiple cells in the longitudinal section, which was consistent with the SEM observations (Fig. 1f).

Stomata are pore-like structures surrounded by two specialized epidermal guard cells and the development of stomata is similar to that of trichomes (Torii 2015). Stoma and trichomes are both derived from epidermal cell differentiation. Notably, we found a large number of stomata occurred on the epidermis of tepals along with trichomes in TR (Fig. 1C). However, there were no stomata on the tepals in TL (Fig. 1d). Therefore, there might be a link or nexus of cell fate between trichomes and stomata presence.

Overview of RNA sequencing and assembly

After high-throughput sequencing, the lily transcriptome sequencing summary was shown in Table S2. For each sample, the number of raw reads varied from 74.66 million $\mathrm{kb}$ to 100.44 million $\mathrm{kb}$. After filtering out poor quality sequences and adapters, 71.05 million kb to 95.59 million kb clean reads were obtained for each library and the highest clean reads ratio was up to $96.90 \%$. Total clean bases ranged from 10.50 $\mathrm{Gb}$ to $14.34 \mathrm{~Gb}$. The Q20 ( $\geq 97.10 \%)$ and Q30 ( $\geq 91.87 \%$ ) suggested that the RNA-seq data were of high quality. Additionally, the transcripts were generated after a series of de novo assembly. The longest transcript of each gene was recognized as the unigene. 277817 unigenes were obtained, with a maximum of $38713 \mathrm{bp}$ and a minimum $301 \mathrm{bp}$ in length. The N50 of transcript and unigene were 901 and 934 respectively, suggesting the transcriptome data could be used for subsequent analysis (Table S3).

Identification of differentially expressed genes related to trichome development

To identify genes that may participate in trichome development, we examined the difference in gene expression between TR and TL in Lilium pumilum. In RNA-seq analysis, the method FPKM (Fragments Per Kilobase of exon model per Million mapped fragments)was used to estimate gene expression level (Trapnell et al. 2010). By pairwise comparisons of TR and TL, a total of 39741 DEGs were identified, of which 10595, 24517, 2896, and 1733 DEGs were found in the comparison of TR1 vs TL1, TL1 vs TL2, TR2 vs TL2, TR1 vs TR2, respectively (Fig. 2a). The number of up-regulated genes was similar to the number of down-regulated genes in each group comparison. In addition, the number of DEGs in the comparison of TR1 vs TL1 was larger than that in TR2 vs TL2, which implied that the genes involved in trichome development may be concentrated in the early stage of flower bud development.

Further, the DEGs were plotted as a Venn diagram and we focused on the 301 unigenes, which were expressed differentially between TR and TL at all stages (Fig. 2b). After the sequence alignment for these 
genes, 107 DEGs that had annotated information were obtained for further analysis. The number of upand down- regulated genes were shown in Fig. 2c. In addition, we drew a heat map of 107 DEGs to display their expression profiles and we conducted hierarchical clustering analysis, as well (Fig. 2d). The heat map showed that the expression patterns of most DEGs between TR and TL were exactly the opposite. Overall, $69.22 \%$ of DEGs had abundant transcripts in TR while low in TL (Fig. 2d).

After checking these 107 DEGs one by one, we screened out several possible trichome-related genes. Meanwhile, we also check the expression pattern of their homologous genes in trichome by using Arabidopsis 'eFP browser'. We identified 27 DEGs and they were classified into four categories: epidermal cell cycle and division, trichome morphogenesis, stress responsiveness, and TFs. All the expression patterns and annotations of the DEGs were shown in the heat map (Fig. 3a-d).

Trichomes are specialized structure on plant epidermis. The epidermal cell division and differentiation are essential to the process of trichome formation (Kryvych et al. 2008). We identified four DEGs possibly related to cell cycle and division. They may be directly involved in the process of cell division (Fig. 3a). With the development of trichomes, many genes associated with biosynthesis and metabolism are expressed with high abundance. In this study, seven DEGs may provide a material basis for the trichome formation such as genes encoding glutamyl-tRNA reductase and pectin acetyl esterase (Fig. 3b). However, three DEGs that were expressed higher in TL may not be conducive to the trichome formation (Fig. 3b). As mentioned above, the trichomes on the plant surface have a great correlation to stresses. We identified seven DEGs possibly involved in response to abiotic stress (Fig. 3c). They were expressed in high abundance in TR except for Cluster-13478.55596. For example, Cluster-13478.101016 was similar to AT3G04880, which encodes a novel protein involved in DNA repair from UV damage. Meanwhile, TFs were also involved in trichome-related processes (Fig. 3d). For example, Cluster-13478.79052 was a putative NAC domain protein 48, expressed lower in TR than that in $T L$, suggesting it may have a negative role in trichome development. Cluster-13478.64633 is homologous to MYB106 in Arabidopsis, which has been shown to have a negative role in trichome branching in Arabidopsis (Jakoby et al. 2008).

GO annotation and KEGG pathway analysis

A total of 105102 unigenes were aligned to GO database, which was grouped into 'biological process/ BP', 'molecular function/ MF', and 'cellular component/ CC' (Fig. S1). In the comparison of TR1 vs TL1, the enriched genes were summarized in the ' $C C^{\prime}$ ' and only 562 genes were enriched in the 'MF'. Surprisingly, no DEGs were enriched in the 'BP'. On the aspect of 'CC', 'intracellular organelle' and 'organelle' were significantly enriched, including 787 and 793 DEGs respectively. To 'MF', 'structural constituent of ribosome' was the most enriched and 151 DEGs were enriched in this process. These results suggest that in the early stage of trichome development, the main differences occur in genes related to cell components and organelles and that may be where structural change starts to occur (Fig. 4a). Compared with DEGs in TR1 vs TL1, fewer genes could be enriched in the comparison of TR2 vs TL2. The most enriching part was 'structural molecular activity', including 62 genes (Fig. 4b). Moreover, there was no other process that contained more than 20 DEGs in 'MF' and no DEG was enriched in the 
'CC' as well. These results suggested that trichome-related activities were less active in the later stages and resulted in a smaller difference between TR2 and TL2 (Fig. 4b). Additionally, we also calculated GO enrichments of the 107 DEGs as mentioned above (Fig. 2d). These 107 DEGs were enriched in the process of cell development and the anabolism of substances, such as 'cellular process', 'metabolic process', 'cell part', and 'membrane' (Fig. 4c).

KEGG pathway was determined to describe the metabolic pathways in cells for DEGs analysis (Kanehisa et al. 2008). A total of 45461 unigenes were enriched and shown in Fig. S2. Here we listed the top 20 of the KEGG pathways enrichment results in comparison of TR1 vs TL1 and TR2 vs TL2 (Fig. 5a, b). Our data showed that 'Ribosome' was most enriched in both comparisons, of which 57 DEGs in TR1 vs TL1 and 30 DEGs in TR2 vs TL2. In addition, four pathways, 'Thiamine metabolism', 'Glycerolipid metabolism', 'Glutathione metabolism', and 'Fructose and mannose metabolism' that are related to substance biosynthesis and metabolism were also significantly enriched in both comparisons. Notably, a total of 9 DEGs in the 'cell cycle' pathway enriched in the comparison between TR vs TL, indicating that those genes may be involved in the cell division of epidermal cells and trichome initiation.

Expression profiling of transcription factors associated with trichome development

Transcription factors are important proteins to regulate gene expression and play critical roles in trichome development (Seo et al. 2013; Liu and Stewart 2016; Wang et al. 2016). As per the RNA-seq data, a total of 6996 transcription factors were identified as shown in Fig S3. After analysis of TFs-related unigenes, we finally selected 249 TFs that expressed differentially between TR and TL. These TFs were mainly classified into 25 families and the number of TFs was shown in Fig. 6. Notably, among 249 TFs, the members of WRKY family, bZIP family, zf-HD family, BBR/BPC family, and TUB family were up-regulated in TR while the members of HSF family, MADS family, ARF family, C2C2-Dof family were down-regulated in TR (Fig. 6). Furthermore, the expression pattern of 141 TFs that were thought to associate with trichome development was also shown in the heat map (Fig. 7a-f, Table. S4-S9).

As previous research showed, the MYB-bHLH-WD40 (MBW) protein complex plays a dominant role in trichome development in Arabidopsis (Wei et al. 2019). Among the trichome-related transcription factor families, the MYB family accounted for the largest percentage (18.4\%), containing 26 up-regulated TFs and 20 down-regulated TFs, followed by bHLH family (12.8\%, 27 up-regulated and 5 down-regulated) (Fig. 7a, b). The WRKY family which consists of the WRKY domain and the $W$ box is also involved in the regulation of trichome development. TRANSPARENT TESTA GLABRA 2 (TTG2) gene was part of WRKY family. In ttg2 mutant, the number of trichomes and their branching is reduced (Pesch et al. 2014). In this study, 10 putative WRKY genes were classified, and their expression levels all increased in TR compared with TL, implying that the TFs might positively regulate trichome development (Fig. 7c). AP2/EREBP transcription factors are related to various physiological and biochemical signals transduction, partially related to stress response (Dietz et al. 2010). In addition, bZIP and NAC are also important transcription factor families involved in the stress response (Jakoby et al. 2002; Singh et al. 2016). In the present study, 32 AP2/EREBP genes, 10 NAC genes, and 8 bZIP genes were identified. Most of them, except for Cluster- 
65226.1, Cluster-13478.108123 and Cluster-13478.169417 were up-regulated in TR compared with TL (Fig. 7d, e, f). The above genes may be involved in the process of trichome-related resistance to biotic and abiotic stress. Interestingly, the expression of most of TFs, as the heatmap showed were significantly different in the early stage (TR1 vs TL1) from the heat map, suggesting that they may be mainly involved in the early stage of trichome development.

Validation of DEGs through qRT-PCR analysis

To verify the authenticity of the RNA-seq data, 14 trichome-related genes were selected for validation by qRT-PCR analysis. These genes contained structural genes, TFs as well as stress response genes. We first confirmed our RNA-seq data by qRT-PCR using Lilium pumilum flower bud at $13.5 \mathrm{~mm}$ and $33.5 \mathrm{~mm}$ in lengths. The expression tendencies of tested genes by qRT-PCR within $13.5 \mathrm{~mm}$ flower buds were consistent with the RNA-seq data (Fig. 8). In addition, the expression tendencies within the later stage were also mostly consistent except Cluster-13478.77595 (Fig S4).

To further identify candidate genes related to trichome development, we also performed qRT-PCR within two Asiatic lily species, i.e. L. lei (trichome) and L.dav (trichomeless). The expression profiles of the tested genes by qRT-PCR were consistent with RNA-seq data (Fig. 9 and S5). Combining the expression profiles of the homologous gene in Arabidopsis by eFP browser, we identify three uncharacterized candidate genes that may have conserved functions in trichome development in lily and Arabidopsis, i.e. ATP-citrate lyase A-3 (ACLA-3), DNA-DAMAGE-REPAIR/TOLERATION 2 (DRT102) and MYB15 (Fig. S5).

\section{Discussion}

Different plant trichome types provide significant model systems for investigating cell differentiation and morphogenesis. In recent years, several genes involved in trichome initiation and development have been investigated in Arabidopsis and tomato. In Arabidopsis, the MYB-bHLH-WD Repeat complex (MBW) regulates trichome development and contains both activators and repressors of trichome development (Pesch et al. 2015; Li et al. 2016). In cotton, two important MADS-box transcription factors, MADS11 and MADS14 have positive roles in trichome initiation and elongation (Li et al. 2011; Zhou et al. 2014). However, less is known in other non-model species such as lily. In this study, we identified several candidate genes for tepal trichome development in Lilium pumilum by transcriptome profiling and qRTPCR, and these genes could be further used in lily breeding.

By SEM and TEM scanning, the trichomes on the tepal in Lilium pumilum was dissected to be the multicellular and branchless type (Fig. 1). Trichomes are widely distributed on the plant surface and they acts as a barrier for plants to resist external stress (Pang et al. 1993). There are some clues that flower-related genes are involved in trichome development. FLOWERING LOCUS C $(F L C)$ that belongs to MADS-box gene can significantly inhibit plant flowering (Michaels and Amasino 1999; Alexandre and Hennig 2008). When this gene is knocked out in Arabidopsis (flc mutant), the number of trichomes is significantly increased (Willmann and Poethig 2011). AGAMOUS (AG) not only regulates the formation of stamen and carpel but also inhibits trichome initiation by regulating cytokinins pathway and cooperating with the organ polarity 
gene KANADI1 (O'Maoileidigh et al. 2018). However, the linkage between trichome and stomata development on the floral organ is still largely unknown.

Ribosomes are mainly composed of rRNA (ribosomal RNA) and ribosomal proteins, and are essential for protein synthesis in cells (Weis et al. 2015). In our results, DEGs was most enriched in 'Ribosome' (Fig. 5). Previous study showed that nuclear ribosome biogenesis mediated by the DIM1A (ADENOSINE DIMETHYL TRANSFERASE 1A) rRNA dimethylase is required for trichome branching in Arabidopsis. In the dim1a mutant, there was a significant decrease in trichome branching (Wieckowski and Schiefelbein 2012). This suggests that ribosome biogenesis is correlated to trichome development, although the mechanism remains to be elucidated. In addition, we also found that many DEGs were enriched in the 'Glutathione metabolism' and 'Fructose and mannose metabolism'. GSH is an important regulatory metabolite in cells and can protect plants from the poison of cadmium and other metals (Schutzendubel and Polle 2002). Soluble sugars such as fructose and mannose can regulate the osmotic pressure of plants and protect them from osmotic stress. For example, the transcription factor AREB2 (ABAresponsive element binding protein 2) in Malus domestica can activate sugar transporter and amylase genes to promoter sugar accumulation (Ma et al. 2017). Considering the positive significance of trichome in plant resistance to abiotic stress, these metabolic pathways may be related to the function of trichome.

Stomata is a specialized structure on the epidermis and the movement is significantly important for plant gas exchange and water use efficiency under drought conditions (Bergmann and Sack 2007; Pillitteri and Torii 2012; Martin-StPaul et al. 2017; Wolz et al. 2017). In the natural mutant, we observed that a large number of stomata were distributed on the epidermis of tepals while no stomata were found on the wildtype tepals (Fig. 1C, d). This correlated phenotype suggested that there may be some genes regulating the fate of both stomata and trichomes. These genes need to be investigated in future studies.

Trichomes differentiate from the epidermal cells after endoreduplications (Pang et al. 1993). The previous study suggested that $A T C D C 48$ encodes a protein involved in the cell division cycle (Park et al. 2008). In the course of our research, the gene sequence of $\angle p C D C 48$ (Cluster-13478.162139) was compared to $A T C D C 48$ and the expression level of $\angle P C D C 48$ was significantly higher in TR than TL, suggesting that it may play a vital role in trichome formation (Fig. 3a). Enzymes are a class of chemical substances with significant catalytic effect, which are widely involved in various material metabolism. We found that eight differently expressed genes were related to enzymes and may play important roles in biosynthesis process (Fig. 3b). For example, LpNIC1(Cluster-13478.72683) whose sequence was similar to NICOTINAMIDASE 1, probably encoded a nicotinamidase to participate in the pyridine nucleotide salvage pathway (Hunt et al. 2007). LpACLA3(Cluster-13478.124788) was expressed higher in TR than TL and was aligned to AT1G09430. The function of AT1G09430 in Arabidopsis is to encode ATP-citrate lyase (ACLY), a bridge between sugar metabolism and fatty acid biosynthesis (Zhao et al. 2016). At the same time, the expression level of AT1G09430 is higher in wild type than that of trichome mutants through the 'eFP-seq browser' (Fig S8 a). As mentioned above, many DEGs were enriched in carbohydrate metabolism and 'Ribosome' pathway, suggesting that precisely regulating genes involved in energy 
supply and protein synthesis is needed for the development of trichomes. These results indicated that LPACLA3 may provide material and energy for the trichome formation in Lilium pumilum.

Lilium pumilum is mainly distributed between the north of China and Qin Mountains, where there is strong UV light in summer. The UV radiation is divided into the UV-A, UV-B, and UV-C range (Krizek and Chalker-Scott 2005). Among them, UV-B radiation (wavelength from $280 \mathrm{~nm}$ to $320 \mathrm{~nm}$ ) makes a great impact on plant growth. It can induce DNA damage and inhibit protein synthesis, resulting in blocking cell division and formation of specific tissues (Mullenders 2018). Plant trichomes, as a natural barrier between plants and the environment, have the function of protecting plants against UV-B radiation damage (Yamasaki et al. 2007). In Arabidopsis, g/1 and g/3 mutants that have a lower trichome density were more vulnerable to UV-B stress than wild type (Yan et al. 2012). Glandular trichomes have glands that secrete flavonoids to absorb ultraviolet light (Gonzales et al. 2015; Huchelmann et al. 2017). Additionally, plants can synthesize DNA-damage-repair/toleration proteins (DRT) against UV light. AtDRT100 encodes a novel protein involved in DNA repair from UV damage (Pang et al. 1993). In grape, overexpression of $V V D R T 100-L$ plants still grew healthy under high-intensity UV light (Fujimori et al. 2014). In this study, we found that $L P D R T 102$ (Cluster-13478.101016) is only expressed in TR (Fig. 3c and Fig. 9). Besides, its homologous gene ATDRT102 has a more abundant transcript level in trichomes than trichomeless tissues in Arabidopsis (Fig. S8b). These results suggest that DRT102 may play important roles in trichome development and UV stress.

The MYB family members mainly act as transcription factors and have functions in a variety of plant processes, such as synthesizing anthocyanins and responding to biotic and abiotic stresses (Stracke et al. 2001; Feller et al. 2011; Li et al. 2019). In addition, several MYB members are involved in leaf trichome initiation and development in Arabidopsis as well (Marks et al. 1991; Wang and Chen 2014). In Arabidopsis, a novel oncogene with a kinase domain (NOK/NOECK) encodes a MIXTA-like transcription factor AtMYB106 and is involved in trichome branching (Trapnell et al. 2010). The nok mutant shows an increased number of branchpoints, suggesting that AtMYB106 protein can inhibit the formation of trichome branches. (Jakoby et al. 2008; Gilding and Marks 2010). In this study, we found that LpMYB106 (Cluster-13478.64633) whose sequence was similar to AtNOK was expressed at a higher level in TR at the early stage (Fig. 3c). In addition, the trichome of Lilium pumilum buds showed no branches by SEM observation. We hypothesized that $\angle p M Y B 106$ may suppress trichome branching in Lilium pumilum. MYB15 is a member of the R2R3 subfamily and is associated with various stress responses (Ding et al. 2009). It has been reported that $A t M Y B 15$ can regulate plant lignification to enhance the basal immunity in Arabidopsis and the promoter of $V V M Y B 15$ also increase pattern-triggered immunity in Chinese wild grape (Chezem et al. 2017; Luo et al. 2019). In tomato, SIMYB15 transcription factors effectively enhanced plant cold tolerance via the C-REPEAT BINDING FACTOR (CBF) pathway, which is conducive to the survival of plants at low temperatures (Zhang et al. 2020). In this study, we identified that $L p M Y B 15$ (Cluster-13478.45016) was aligned to AtMYB15, and expressed higher in TR than TL at all stages. At the same time, AtMYB15 was expressed higher in wild-type and g/3-sst sim mutant with trichome than other mutants (Fig S8 c). That meant Cluster-13478.45016 may have the same function as AtMYB15 to stress resistance. 
In conclusion, we found a natural mutant of Lilium pumilum whose tepals are covered by multicellularand branchless- trichomes. Transcriptome analysis revealed several genes involved in epidermal cell cycle and division, trichome morphogenesis, stress response, and transcription factors which may possibly regulate trichome development in Lilium pumilum. Quantitative real-time PCR in Lilium pumilum and other lily species (Lilium leichtlinii var. maximowiczii and Lilium davidii var. willmottiae) confirmed the transcriptome data and identified several trichome- related genes.

\section{Declarations}

\section{Acknowledgments}

This work was funded by Beijing Natural Science Foundation (grants 6212012 to JW), The special projects for capacity-building in scientific and technological innovation of Beijing Academy of Agriculture and Forestry (KJCX20200103), National Natural Science Foundation projects (grants 31601781 to YPD; 31701952 to JW), The Youth Fund Project of Beijing Academy of Agriculture and Forestry (QNJJ201910), Construction of Beijing Science and Technology Innovation and Service Capacity in Top Subjects (CEFFPXM2019_014207_000032) and 111 Project of the Ministry of Education (B17043).

\section{Author contribution}

Y. X., W. P. and C. X. performed the most experiments and analysis in this study; M. Z., X. C., F. Y. and J. L. assisted with the data analysis and plant materials; J. W., Y. D., and X. Z. conceived the study; J. W., W. P. and Y. X. wrote the article; all authors read and approved the article.

\section{Conflict of interest}

The authors declare that they have no conflict of interest.

\section{References}

1. Airoldi CA, Hearn TJ, Brockington SF, Webb AAR, Glover BJ (2019) TTG1 proteins regulate circadian activity as well as epidermal cell fate and pigmentation. Nat Plants 5(11):1145-1153. doi:10.1038/s41477-019-0544-3

2. Alexandre CM, Hennig L (2008) FLC or not FLC: the other side of vernalization. J Exp Bot 59(6):1127-1135. doi:10.1093/jxb/ern070

3. Bac-Molenaar JA, Mol S, Verlaan MG, van Elven J, Kim HK, Klinkhamer PGL, Leiss KA, Vrieling K (2019) Trichome Independent Resistance against Western Flower Thrips in Tomato. Plant Cell Physiol 60(5):1011-1024. doi:10.1093/pcp/pcz018

4. Bergmann DC, Sack FD (2007) Stomatal development. Annu Rev Plant Biol 58:163-181. doi:10.1146/annurev.arplant.58.032806.104023 
5. Bloomer RH, Juenger TE, Symonds VV (2012) Natural variation in GL1 and its effects on trichome density in Arabidopsis thaliana. Mol Ecol 21(14):3501-3515. doi:10.1111/j.1365-294X.2012.05630.X

6. Chen CH, Liu ML, Jiang L, Liu XF, Zhao JY, Yan SS, Yang S, Ren HZ, Liu RY, Zhang XL (2014) Transcriptome profiling reveals roles of meristem regulators and polarity genes during fruit trichome development in cucumber (Cucumis sativus L.). J Exp Bot 65(17):4943-4958. doi:10.1093/jxb/eru258

7. Chezem WR, Memon A, Li FS, Weng JK, Clay NK (2017) SG2-Type R2R3-MYB Transcription Factor MYB15 Controls Defense-Induced Lignification and Basal Immunity in Arabidopsis. Plant Cell 29(8):1907-1926. doi:10.1105/tpc.16.00954

8. Dietz KJ, Vogel MO, Viehhauser A (2010) AP2/EREBP transcription factors are part of gene regulatory networks and integrate metabolic, hormonal and environmental signals in stress acclimation and retrograde signalling. Protoplasma 245(1-4):3-14. doi:10.1007/s00709-010-0142-8

9. Ding ZH, Li SM, An XL, Liu XJ, Qin HM, Wang D (2009) Transgenic expression of MYB15 confers enhanced sensitivity to abscisic acid and improved drought tolerance in Arabidopsis thaliana. $\mathrm{J}$ Genet Genomics 36(1):17-29. Doi 10.1016/S1673-8527(09)60003-5 doi

10. Doroshkov AV, Konstantinov DK, Afonnikov DA, Gunbin KV (2019) The evolution of gene regulatory networks controlling Arabidopsis thaliana L. trichome development. BMC Plant Biol 19:53. doi:10.1186/s12870-019-1640-2

11. Escobar-Bravo R, Klinkhamer PGL, Leiss KA (2017) Induction of Jasmonic Acid-Associated Defenses by Thrips Alters Host Suitability for Conspecifics and Correlates with Increased Trichome Densities in Tomato. Plant Cell Physiol 58(3):622-634. doi:10.1093/pcp/pcx014

12. Feller A, Machemer K, Braun EL, Grotewold E (2011) Evolutionary and comparative analysis of MYB and bHLH plant transcription factors. Plant J 66(1):94-116. doi:10.1111/j.1365-313X.2010.04459.X

13. Fujimori N, Suzuki N, Nakajima Y, Suzuki S (2014) Plant DNA-damage repair/toleration 100 protein repairs UV-B-induced DNA damage. DNA Repair 21:171-176. doi:10.1016/j.dnarep.2014.05.009

14. Gilding EK, Marks MD (2010) Analysis of purified glabra3-shapeshifter trichomes reveals a role for NOECK in regulating early trichome morphogenic events. Plant J 64(2):304-317. doi:10.1111/j.1365313X.2010.04329.x

15. Gonzales GB, Smagghe G, Grootaert C, Zotti M, Raes K, Van Camp J (2015) Flavonoid interactions during digestion, absorption, distribution and metabolism: a sequential structure-activity/property relationship-based approach in the study of bioavailability and bioactivity. Drug Metab Rev 47(2):175-190. doi:10.3109/03602532.2014.1003649

16. Grabherr MG, Haas BJ, Yassour M, Levin JZ, Thompson DA, Amit I, Adiconis X, Fan L, Raychowdhury R, Zeng QD, Chen ZH, Mauceli E, Hacohen N, Gnirke A, Rhind N, di Palma F, Birren BW, Nusbaum C, Lindblad-Toh K, Friedman N, Regev A (2011) Full-length transcriptome assembly from RNA-Seq data without a reference genome. Nat Biotechnol 29(7):644-130. doi:10.1038/nbt.1883

17. Huchelmann A, Boutry M, Hachez C (2017) Plant Glandular Trichomes: Natural Cell Factories of High Biotechnological Interest. Plant Physiol 175(1):6-22. doi:10.1104/pp.17.00727 
18. Hulskamp M (2004) Plant trichomes: a model for cell differentiation. Nat Rev Mol Cell Biol 5(6):471480. doi: $10.1038 / \mathrm{nrm} 1404$

19. Hunt L, Holdsworth MJ, Gray JE (2007) Nicotinamidase activity is important for germination. Plant J 51(3):341-351. doi:10.1111/j.1365-313X.2007.03151.x

20. Jakoby M, Weisshaar B, Droge-Laser W, Vicente-Carbajosa J, Tiedemann J, Kroj T, Parcy F, Grp bR (2002) bZIP transcription factors in Arabidopsis. Trends Plant Sci 7(3):106-111. doi:10.1016/S13601385(01)02223-3

21. Jakoby MJ, Falkenhan D, Mader MT, Brininstool G, Wischnitzki E, Platz N, Hudson A, Lskamp MHR, Larkin J, Schnittger A (2008) Transcriptional Profiling of Mature Arabidopsis Trichomes Reveals That NOECK Encodes the MIXTA-Like Transcriptional Regulator MYB106. Plant Physiol 148(3):15831602. doi:10.1104/pp.108.126979

22. Kanehisa M, Araki M, Goto S, Hattori M, Hirakawa M, Itoh M, Katayama T, Kawashima S, Okuda S, Tokimatsu T, Yamanishi Y (2008) KEGG for linking genomes to life and the environment. Nucleic Acids Res 36:D480-D484. doi:10.1093/nar/gkm882

23. Khosla A, Paper JM, Boehler AP, Bradley AM, Neumann TR, Schrick K (2014) HD-Zip Proteins GL2 and HDG11 Have Redundant Functions in Arabidopsis Trichomes, and GL2 Activates a Positive Feedback Loop via MYB23. Plant Cell 26(5):2184-2200. doi:10.1105/tpc.113.120360

24. Kirik V, Simon M, Huelskamp M, Schiefelbein J (2004) The ENHANCER OF TRY AND CPC1 gene acts redundantly with TRIPTYCHON and CAPRICE in trichome and root hair cell patterning in Arabidopsis. Dev Biol 268(2):506-513. doi:10.1016/j.ydbio.2003.12.037

25. Krizek DT, Chalker-Scott L (2005) Ultraviolet radiation and terrestrial ecosystems. Photochem Photobiol 81(5):1021-1025. doi:10.1562/2005-08-18-Ra-654

26. Kryvych S, Nikiforova V, Herzog M, Perazza D, Fisahn J (2008) Gene expression profiling of the different stages of Arabidopsis thaliana trichome development on the single cell level. Plant Physiol Bioch 46(2):160-173. doi:10.1016/j.plaphy.2007.11.001

27. Li JL, Han GL, Sun CF, Sui N (2019) Research advances of MYB transcription factors in plant stress resistance and breeding. Plant Signal Behav 14(8):1613131. doi:10.1080/15592324.2019.1613131

28. Li QY, Yin M, Li YP, Fan CC, Yang QY, Wu J, Zhang CY, Wang H, Zhou YM (2015a) Expression of Brassica napus TTG2, a regulator of trichome development, increases plant sensitivity to salt stress by suppressing the expression of auxin biosynthesis genes. J Exp Bot 66(19):5821-5836. doi:10.1093/jxb/erv287

29. Li X, Cheng J, Zhang J, Teixeira da Silva JA, Wang C, Sun H (2015b) Validation of Reference Genes for Accurate Normalization of Gene Expression in Lilium davidii var. unicolor for Real Time Quantitative PCR. Plos One 10(10):e0141323. doi:10.1371/journal.pone.0141323

30. Li Y, Ning H, Zhang ZT, Wu Y, Jiang J, Su SY, Tian FY, Li XB (2011) A cotton gene encoding novel MADS-box protein is preferentially expressed in fibers and functions in cell elongation. Acta Bioch Bioph Sin 43(8):607-617. doi:10.1093/abbs/gmr055 
31. Li YQ, Shan XT, Gao RF, Yang S, Wang SC, Gao X, Wang L (2016) Two Illf Clade-bHLHs from Freesia hybrida Play Divergent Roles in Flavonoid Biosynthesis and Trichome Formation when Ectopically Expressed in Arabidopsis. Sci Rep 6:30514. doi:10.1038/srep30514

32. Liu WS, Stewart CN (2016) Plant synthetic promoters and transcription factors. Curr Opin Biotech 38:203-203. doi:10.1016/j.copbio.2016.03.017

33. Livingston SJ, Quilichini TD, Booth JK, Wong DCJ, Rensing KH, Laflamme-Yonkman J, Castellarin SD, Bohlmann J, Page JE, Samuels AL (2020) Cannabis glandular trichomes alter morphology and metabolite content during flower maturation. Plant J 101(1):37-56. doi:10.1111/tpj.14516

34. Luo YY, Bai R, Li J, Yang WD, Li RX, Wang QY, Zhao GF, Duan D (2019) The transcription factor MYB15 is essential for basal immunity (PTI) in Chinese wild grape. Planta 249(6):1889-1902. doi:10.1007/s00425-019-03130-5

35. Ma D, Hu Y, Yang C, Liu B, Fang L, Wan Q, Liang W, Mei G, Wang L, Wang H, Ding L, Dong C, Pan M, Chen J, Wang S, Chen S, Cai C, Zhu X, Guan X, Zhou B, Zhu S, Wang J, Guo W, Chen X, Zhang T (2016) Genetic basis for glandular trichome formation in cotton. Nat Commun 7:10456. doi:10.1038/ncomms10456

36. Ma QJ, Sun MH, Lu J, Liu YJ, Hu DG, Hao YJ (2017) Transcription Factor AREB2 Is Involved in Soluble Sugar Accumulation by Activating Sugar Transporter and Amylase Genes. Plant Physiol 174(4):2348-2362. doi:10.1104/pp.17.00502

37. Mao XZ, Cai T, Olyarchuk JG, Wei LP (2005) Automated genome annotation and pathway identification using the KEGG Orthology (KO) as a controlled vocabulary. Bioinformatics 21(19):3787-3793. doi:10.1093/bioinformatics/bti430

38. Marks MD, Esch J, Herman P, Sivakumaran S, Oppenheimer D (1991) A model for cell-type determination and differentiation in plants. Symp Soc Exp Biol 45:77-87

39. Martin-StPaul N, Delzon S, Cochard H (2017) Plant resistance to drought depends on timely stomatal closure. Ecology letters 20(11):1437-1447. doi:10.1111/ele.12851

40. Michaels SD, Amasino RM (1999) FLOWERING LOCUS C encodes a novel MADS domain protein that acts as a repressor of flowering. Plant Cell 11(5):949-956. doi:10.1105/tpc.11.5.949

41. Mitteer DR, Greer BD, Randall KR, Briggs AM (2020) Further Evaluation of Teaching Behavior Technicians to Input Data and Graph Using GraphPad Prism Behav Anal (Wash D C) 20 (2): 81-93. doi:10.1037/bar0000172

42. Morohashi K, Zhao M, Yang M, Read B, Lloyd A, Lamb R, Grotewold E (2007) Participation of the Arabidopsis bHLH factor GL3 in trichome initiation regulatory events. Plant Physiol 145(3):736-746. doi:10.1104/pp.107.104521

43. Mullenders LHF (2018) Solar UV damage to cellular DNA: from mechanisms to biological effects. Photoch Photobio Sci 17(12):1842-1852. doi:10.1039/c8pp00182k

44. Nachamkin I, Panaro NJ, Li M, Ung H, Yuen PK, Kricka LJ, Wilding P (2001) Agilent 2100 bioanalyzer for restriction fragment length polymorphism analysis of the Campylobacter jejuni flagellin gene. $\mathrm{J}$ Clin Microbiol 39(2):754-757. doi:10.1128/JCM.39.2.754-757.2001 
45. O'Maoileidigh DS, Stewart D, Zheng B, Coupland G, Wellmer F (2018) Floral homeotic proteins modulate the genetic program for leaf development to suppress trichome formation in flowers. Development 145(3):dev157784. doi:10.1242/dev.157784

46. Okamoto S, Negishi K, Toyama Y, Ushijima T, Morohashi K (2020) Leaf Trichome Distribution Pattern in Arabidopsis Reveals Gene Expression Variation Associated with Environmental Adaptation. Plants (Basel) 9(7):909. doi:10.3390/plants9070909

47. Ozturk Cali I, Karavin N (2020) Influence of auto-exhaust pollution on trichome micromorphology of Olea europea L. leaves. Microsc Res Tech 83(5):558-561. doi:10.1002/jemt.23445

48. Pang QS, Hays JB, Rajagopal I, Schaefer TS (1993) Selection of Arabidopsis Cdnas That Partially Correct Phenotypes of Escherichia-Coli DNA-Damage-Sensitive Mutants and Analysis of 2 Plant Cdnas That Appear To Express Uv-Specific Dark Repair Activities. Plant Mol Biol 22(3):411-426. doi:10.1007/Bf00015972

49. Park S, Rancour DM, Bednarek SY (2008) In planta analysis of the cell cycle-dependent localization of AtCDC48A and its critical roles in cell division, expansion, and differentiation. Plant Physiol 148(1):246-258. doi:10.1104/pp.108.121897

50. Pesch M, Dartan B, Birkenbihl R, Somssich IE, Hulskamp M (2014) Arabidopsis TTG2 Regulates TRY Expression through Enhancement of Activator Complex-Triggered Activation. Plant Cell 26(10):4067-4083. doi:10.1105/tpc.114.129379

51. Pesch M, Schultheiss I, Klopffleisch K, Uhrig JF, Koegl M, Clemen CS, Simon R, Weidtkamp-Peters S, Hulskamp M (2015) TRANSPARENT TESTA GLABRA1 and GLABRA1 Compete for Binding to GLABRA3 in Arabidopsis. Plant Physiol 168(2):584-597. doi:10.1104/pp.15.00328

52. Pillitteri LJ, Torii KU (2012) Mechanisms of stomatal development. Annu Rev Plant Biol 63:591-614. doi:10.1146/annurev-arplant-042811-105451

53. Schutzendubel A, Polle A (2002) Plant responses to abiotic stresses: heavy metal-induced oxidative stress and protection by mycorrhization. J Exp Bot 53(372):1351-1365

54. Seale M, Cummins C, Viola IM, Mastropaolo E, Nakayama N (2018) Design principles of hair-like structures as biological machines. J R Soc Interface 15(142):20180206. doi:10.1098/rsif.2018.0206

55. Seo PJ, Park MJ, Park CM (2013) Alternative splicing of transcription factors in plant responses to low temperature stress: mechanisms and functions. Planta 237(6):1415-1424. doi:10.1007/s00425013-1882-4

56. Singh S, Grover A, Nasim M (2016) Biofuel Potential of Plants Transformed Genetically with NAC Family Genes. Front Plant Sci 7:22. doi:10.3389/fpls.2016.00022

57. Stracke R, Werber M, Weisshaar B (2001) The R2R3-MYB gene family in Arabidopsis thaliana. Curr Opin Plant Biol 4(5):447-456. doi:10.1016/S1369-5266(00)00199-0

58. Sullivan A, Purohit PK, Freese NH, Pasha A, Esteban E, Waese J, Wu A, Chen M, Chin CY, Song R, Watharkar SR, Chan AP, Krishnakumar V, Vaughn MW, Town C, Loraine AE, Provart NJ (2019) An 'eFPSeq Browser' for visualizing and exploring RNA sequencing data. Plant J 100(3):641-654. doi:10.1111/tpj.14468 
59. Tang T, Li CH, Li DS, Jing SX, Hua J, Luo SH, Liu Y, Li SH (2020) Peltate glandular trichomes of Colquhounia vestita harbor diterpenoid acids that contribute to plant adaptation to UV radiation and cold stresses. Phytochemistry 172:112285. doi:10.1016/j.phytochem.2020.112285

60. Tominaga-Wada R, Nukumizu Y, Sato S, Wada T (2013) Control of Plant Trichome and Root-Hair Development by a Tomato (Solanum lycopersicum) R3 MYB Transcription Factor. Plos One 8(1):e54019. doi:10.1371/journal.pone.0054019

61. Torii KU (2015) Stomatal differentiation: the beginning and the end. Curr Opin Plant Biol 28:16-22. doi:10.1016/j.pbi.2015.08.005

62. Trapnell C, Williams BA, Pertea G, Mortazavi A, Kwan G, van Baren MJ, Salzberg SL, Wold BJ, Pachter L (2010) Transcript assembly and quantification by RNA-Seq reveals unannotated transcripts and isoform switching during cell differentiation. Nat Biotechnol 28(5):511-515. doi:10.1038/nbt.1621

63. Vadde BVL, Challa KR, Sunkara P, Hegde AS, Nath U (2019) The TCP4 Transcription Factor Directly Activates TRICHOMELESS1 and 2 and Suppresses Trichome Initiation. Plant Physiol 181(4):15871599. doi:10.1104/pp.19.00197

64. Wang S, Chen JG (2014) Regulation of cell fate determination by single-repeat R3 MYB transcription factors in Arabidopsis. Front Plant Sci 5:133. doi:10.3389/fpls.2014.00133

65. Wang Y, Chen W, Qin P, Huang Y, Ma B, Ouyang X, Chen X, Li S (2013) Characterization and fine mapping of Glabrous rice 2 in rice. J Genet Genomics 40(11):579-582. doi:10.1016/j.jgg.2013.06.001

66. Wang YJ, Lu WJ, Deng DX (2016) Bioinformatic landscapes for plant transcription factor system research. Planta 243(2):297-304. doi:10.1007/s00425-015-2453-7

67. Wang Z, Yang Z, Li F (2019) Updates on molecular mechanisms in the development of branched trichome in Arabidopsis and nonbranched in cotton. Plant Biotechnol J 17(9):1706-1722. doi:10.1111/pbi.13167

68. Wei ZL, Cheng YL, Zhou CC, Li D, Gao X, Zhang SX, Chen MX (2019) Genome-Wide Identification of Direct Targets of the TTG1-bHLH-MYB Complex in Regulating Trichome Formation and Flavonoid Accumulation in Arabidopsis Thaliana. Int J Mol Sci 20(20):5014. doi:10.3390/ijms20205014

69. Weis BL, Kovacevic J, Missbach S, Schleiff E (2015) Plant-Specific Features of Ribosome Biogenesis. Trends Plant Sci 20(11):729-740. doi:10.1016/j.tplants.2015.07.003

70. Wieckowski Y, Schiefelbein J (2012) Nuclear ribosome biogenesis mediated by the DIM1A rRNA dimethylase is required for organized root growth and epidermal patterning in Arabidopsis. Plant Cell 24(7):2839-2856. doi:10.1105/tpc.112.101022

71. Willmann MR, Poethig RS (2011) The effect of the floral repressor FLC on the timing and progression of vegetative phase change in Arabidopsis. Development 138(4):677-685. doi:10.1242/dev.057448

72. Wolz KJ, Wertin TM, Abordo M, Wang D, Leakey ADB (2017) Diversity in stomatal function is integral to modelling plant carbon and water fluxes. Nat Ecol Evol 1(9):1292-1298. doi:10.1038/s41559-0170238-z 
73. Wu Z, Liang J, Wang C, Ding L, Zhao X, Cao X, Xu S, Teng N, Yi M (2019) Alternative Splicing Provides a Mechanism to Regulate LIHSFA3 Function in Response to Heat Stress in Lily. Plant Physiol 181(4):1651-1667. doi:10.1104/pp.19.00839

74. Yamasaki S, Noguchi N, Mimaki K (2007) Continuous UV-B irradiation induces morphological changes and the accumulation of polyphenolic compounds on the surface of cucumber cotyledons. J Radiat Res 48(6):443-454. doi:10.1269/jrr.07046

75. Yan A, Pan J, An L, Gan Y, Feng H (2012) The responses of trichome mutants to enhanced ultravioletB radiation in Arabidopsis thaliana. J Photochem Photobiol B 113:29-35. doi:10.1016/j.jphotobiol.2012.04.011

76. Yanagisawa M, Desyatova AS, Belteton SA, Mallery EL, Turner JA, Szymanski DB (2015) Patterning mechanisms of cytoskeletal and cell wall systems during leaf trichome morphogenesis. Nat Plants 1(3):15014. doi:10.1038/Nplants.2015.14

77. Yang CX, Ye ZB (2013) Trichomes as models for studying plant cell differentiation. Cell Mol Life Sci 70(11):1937-1948. doi:10.1007/s00018-012-1147-6

78. Zhang L, Jiang X, Liu Q, Ahammed GJ, Lin R, Wang L, Shao S, Yu J, Zhou Y (2020) The HY5 and MYB15 transcription factors positively regulate cold tolerance in tomato via the CBF pathway. Plant Cell Environ 43(11):2712-2726. doi:10.1111/pce.13868

79. Zhang N, Yang L, Luo S, Wang XT, Wang W, Cheng YX, Tian HN, Zheng KJ, Cai L, Wang SC (2018) Genetic evidence suggests that GIS functions downstream of TCL1 to regulate trichome formation in Arabidopsis. BMC Plant Biol 18:63. doi:10.1186/s12870-018-1271-z

80. Zhao S, Torres A, Henry RA, Trefely S, Wallace M, Lee JV, Carrer A, Sengupta A, Campbell SL, Kuo YM, Frey AJ, Meurs N, Viola JM, Blair IA, Weljie AM, Metallo CM, Snyder NW, Andrews AJ, Wellen KE (2016) ATP-Citrate Lyase Controls a Glucose-to-Acetate Metabolic Switch. Cell Rep 17(4):10371052. doi:10.1016/j.celrep.2016.09.069

81. Zheng KJ, Tian HN, Hu QN, Guo HY, Yang L, Cai L, Wang XT, Liu B, Wang SC (2016) Ectopic expression of R3 MYB transcription factor gene OsTCL1 in Arabidopsis, but not rice, affects trichome and root hair formation. Sci Rep 6:19254. doi:10.1038/srep19254

82. Zhou Y, Li BY, Li M, Li XJ, Zhang ZT, Li Y, Li XB (2014) A MADS-box gene is specifically expressed in fibers of cotton (Gossypium hirsutum) and influences plant growth of transgenic Arabidopsis in a GA-dependent manner. Plant Physiol Bioch 75:70-79. doi:10.1016/j.plaphy.2013.12.003

83. Zhou Z, Tan H, Li Q, Li Q, Wang Y, Bu Q, Li Y, Wu Y, Chen W, Zhang L (2020) TRICHOME AND ARTEMISININ REGULATOR 2 positively regulates trichome development and artemisinin biosynthesis in Artemisia annua. New Phytol 228(3):932-945. doi:10.1111/nph.16777

\section{Figures}



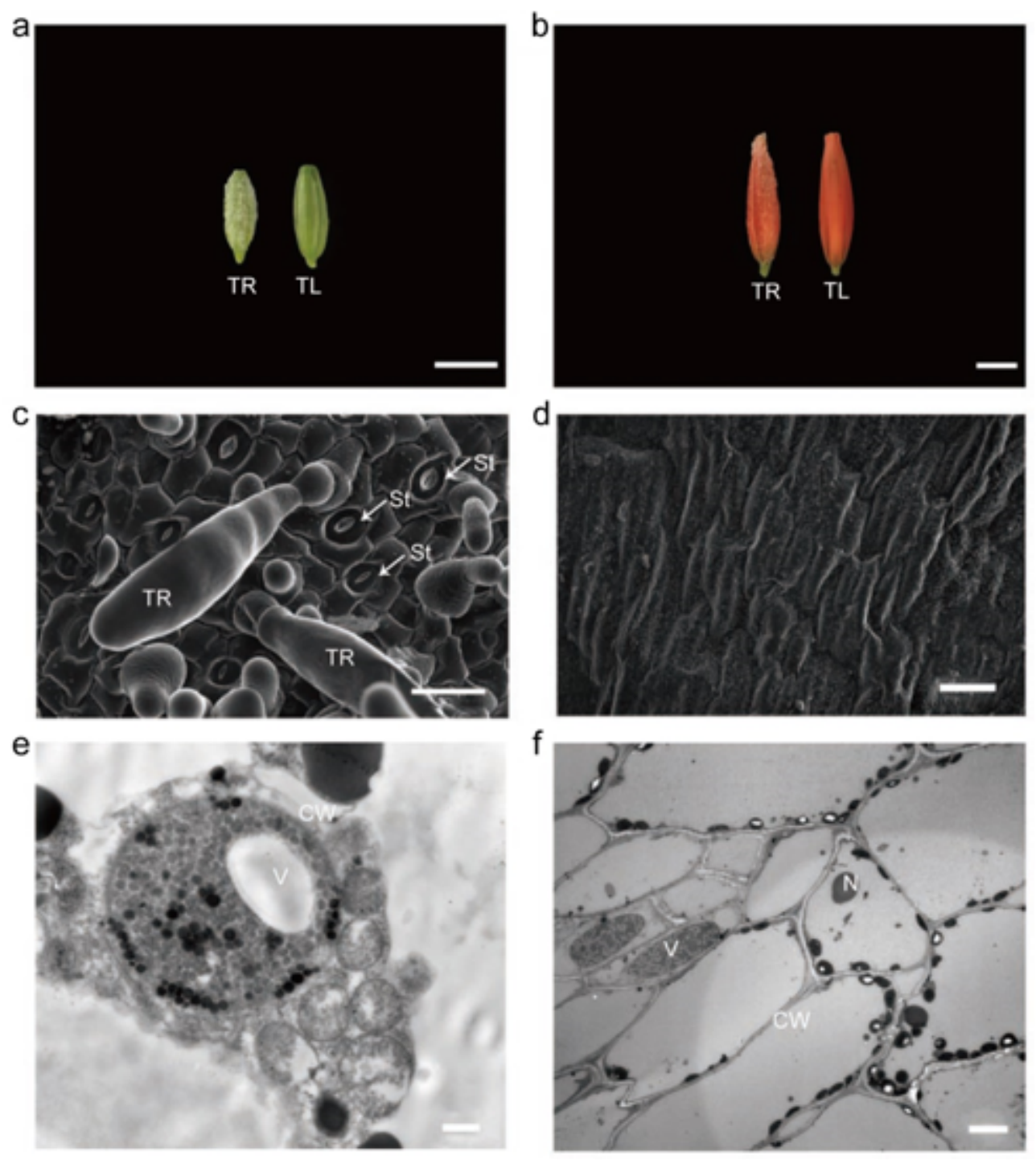

\section{Figure 1}

Scanning of tepal epidermis in L. pumilum by TEM and SEM. Tepals of flowers at two different stages were used for scanning and RNA sequencing. Tepals were green in $13.5 \mathrm{~mm}$ length (a) and coloring in $33.5 \mathrm{~mm}$ in length (b); c Rod-like trichomes and stomata were differentiated on the tepal epidermis of the mutant (TR); d No additional specialized structures formed on the tepal epidermis of TL; The crosssection (e) and longitudinal section (f) of trichome on TR's tepal by TEM. Scale bar in a, b represents 10 $\mathrm{mm}$; scale bar in c, e, f represents $100 \mu \mathrm{m}$; scale bar in d represents $10 \mu \mathrm{m}$. CW, cell wall; N, nucleus; St, stomata; TL, trichomeless; TR, trichome; V, vacuole. 
a

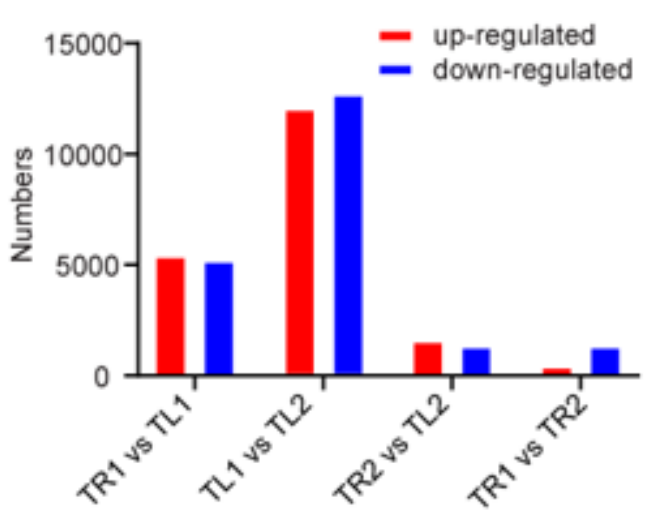

b

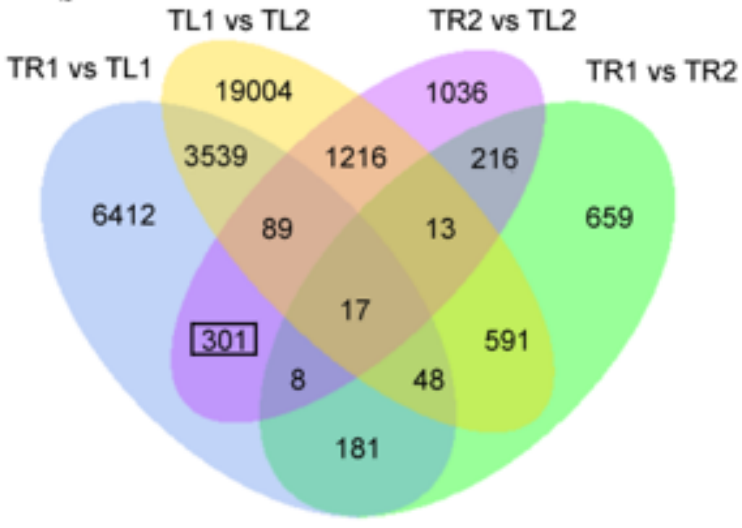

C

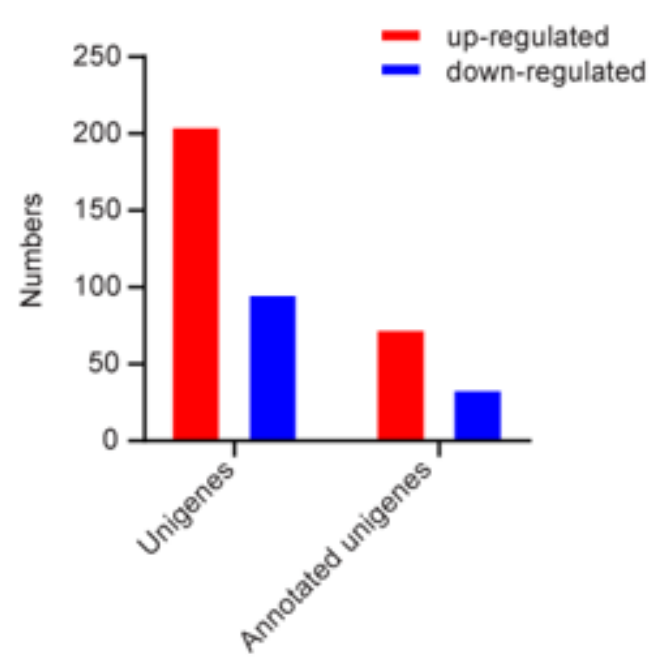

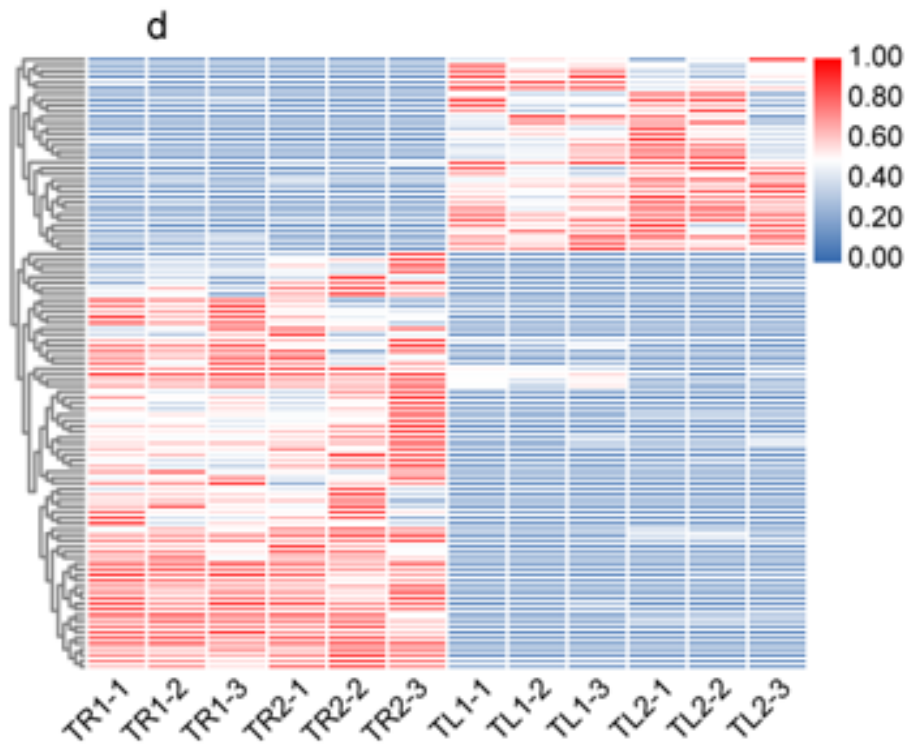

Figure 2

DEGs between TR and TL. a Numbers of DEGs in pairwise comparisons; $b$ Venn diagram showed the number of DEGs in different comparisons; c Numbers of DEGs potentially related to trichome development; $d$ Heatmap diagrams showed the relative expression levels of 107 annotated DEGs. The color scale on the right from blue to red represents FPKM values from low to high. TL, trichomeless; TR, trichome. 
a
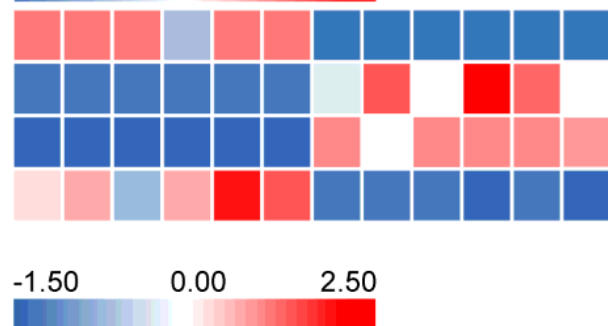

b

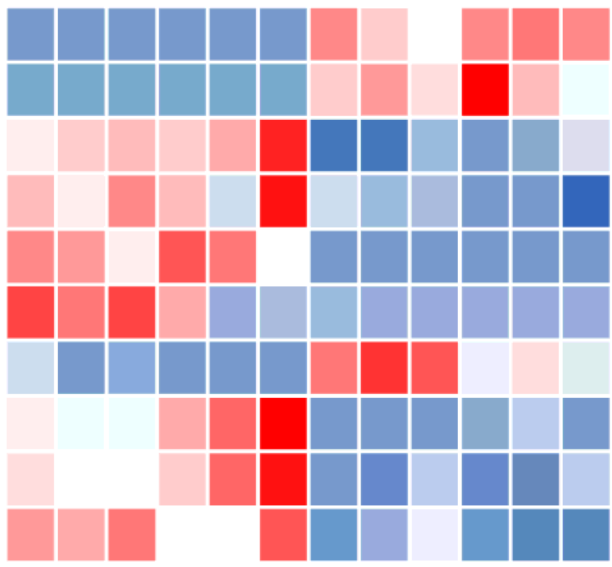

Cluster-13478.75880

Cluster-13478.123455

Cluster-13478.124788

Cluster-13478.89013

Cluster-13478.72683

Cluster-13478.80879

Cluster-13478.63838

Cluster-13478.85388

Cluster-13478.97853

Cluster-13478.19147

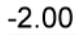

0.00

1.50

C

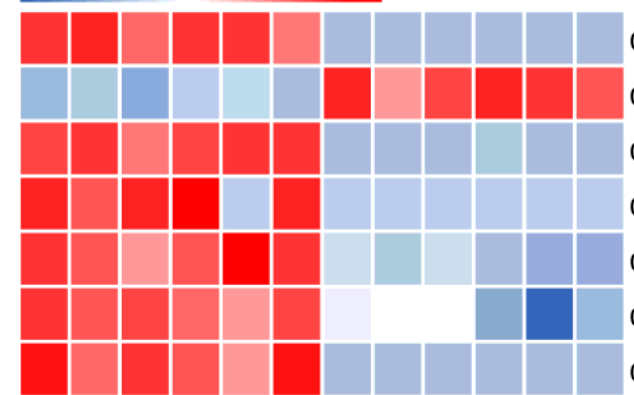

Cluster-13478.87316

Cluster-13478.55596

Cluster-13478.79263

Cluster-13478.101016

Cluster-13478.110406

Cluster-13478.110400

Cluster-13478.79263
Outer envelope protein 80

Chromatin structure-remodeling complex protein SYD

Protein SPIRAL1

Cell division cycle protein 48

Pectin acetylesterase 8

ATP-dependent 6-phosphofructokinase 5

ATP-citrate synthase alpha chain protein 3

Linoleate 9S-lipoxygenase 5

Nicotinamidase 1

Actin-interacting protein 1

Glutamyl-tRNA reductase 2

Thiamine thiazole synthase

Plastid-lipid-associated protein 13

V-type proton ATPase subunit E1

RGG repeats nuclear RNA binding protein $A$

Protein Pop3

SPFH/Band 7/PHB domain-containing protein

DNA-damage-repair/toleration protein DRT102

Stress-response A/B barrel domain-containing protein

60S ribosomal protein L36-3

Protein PPLZ12

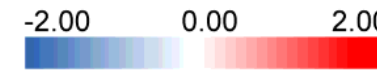

d

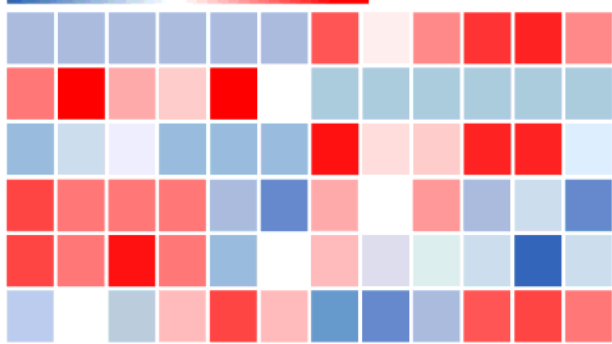

Cluster-13478.79052 NAC domain-containing protein 48

Cluster-13478.48782

GTPase activating protein for ARFdomain

Cluster-13478.150409 ARF-GAP domain-containing protein AGD4

Cluster-13478.64633

Myb-related protein 106

Cluster-13478.45016

Myb-related protein 15

Cluster-13478.64633 Myb-related protein Hv1

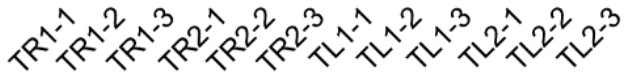

\section{Figure 3}

The heatmap of candidate DEGs involved in the trichome development. a Epidermal cell cycle and division related genes; $b$ Trichome morphogenesis related genes; c Trichome related stress-response genes; $d$ Trichome related transcription factors. The color scale on the top from blue to red represents FPKM values from low to high. 


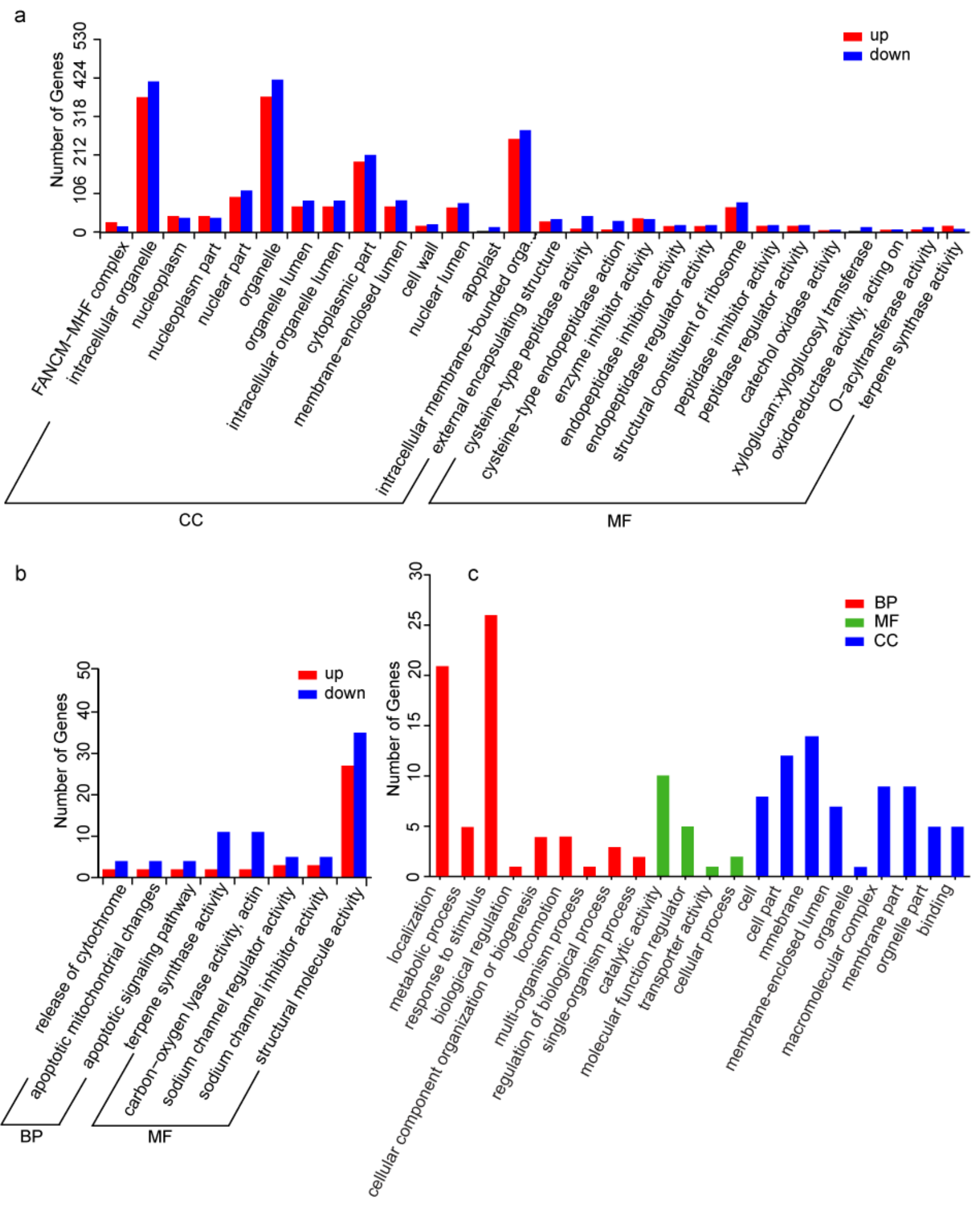

Figure 4

GO classification of unigenes. a Enhanced GO terms in TR1 vs TL1; $b$ Enhanced GO terms in TR2 vs TL2; c Enhanced GO terms in 107 specific DEGs. BP, biological process; MF, molecular function; CC, cellular component. 
a

TR1 vs TL1

Statistics of Pathway Enrichment

Tropane \& pyridine alkaloid biosynthesis

Thiamine metabolism Spliceosome Ribosome

Protein in endoplasmic reticulum

Proteasome

Photosynthesis

Phenylpropanoid biosynthesis

Phenylalanine metabolism

Phagosome

Monoterpenoid biosynthesis Linoleic acid metabolism Isoquinoline alkaloid biosynthesis Glycerolipid metabolism. Glutathione metabolism

Fructose and mannose metabolism

Flavonoid biosynthesis Endocytosis

Cyanoamino acid metabolism Cell cycle - Caulobacter

b

TR2 vs TL2

Statistics of Pathway Enrichment

Thiamine metabolism Sphingolipid metabolism Sesquiterpenoid \&triterpenoid biosynthesis

Regulation of autophagy Protein in endoplasmic reticulum Monoterpenoid biosynthesis Glycerolipid metabolism Glutathione metabolism Galactose metabolism Fructose and mannose metabolism Cutin, suberine and wax biosynthesis. Citrate cycle (TCA cycle)

Cell cycle - Caulobacter beta-Alanine metabolism. Ascorbate and aldarate metabolism. Arginine and proline metabolism Amino sugar \& nucleotide sugar metabolism

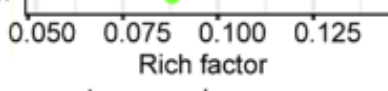

Gene_number qvalue

$50 \bigcirc 100$ qvalue

$\begin{array}{lllll}1.00 & 0.75 & 0.50 & 0.25 & 0.00\end{array}$
Sulfur relay system Ribosome Proteasome

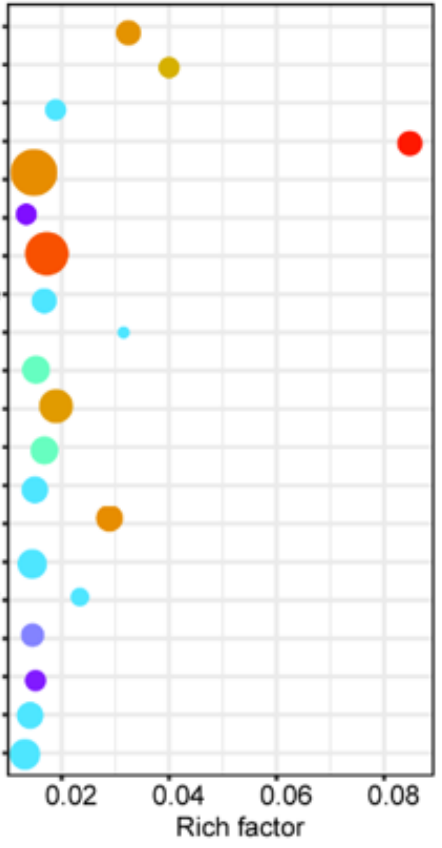

Gene_number

$10 \bigcirc 20$ qvalue

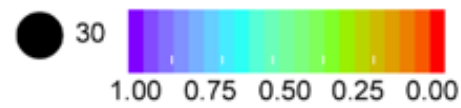

\section{Figure 5}

The top 20 enriched KEGG pathways among the DEGs. a Enriched KEGG pathways in comparison of TR1 vs TL1; $b$ Enriched KEGG pathways in TR2 vs TL2. The y-axis presents the different KEGG pathways and the $\mathrm{x}$-axis presents the rich factor. Dot color means the q-value and dot size represents gene number enriched in the corresponding pathway. 


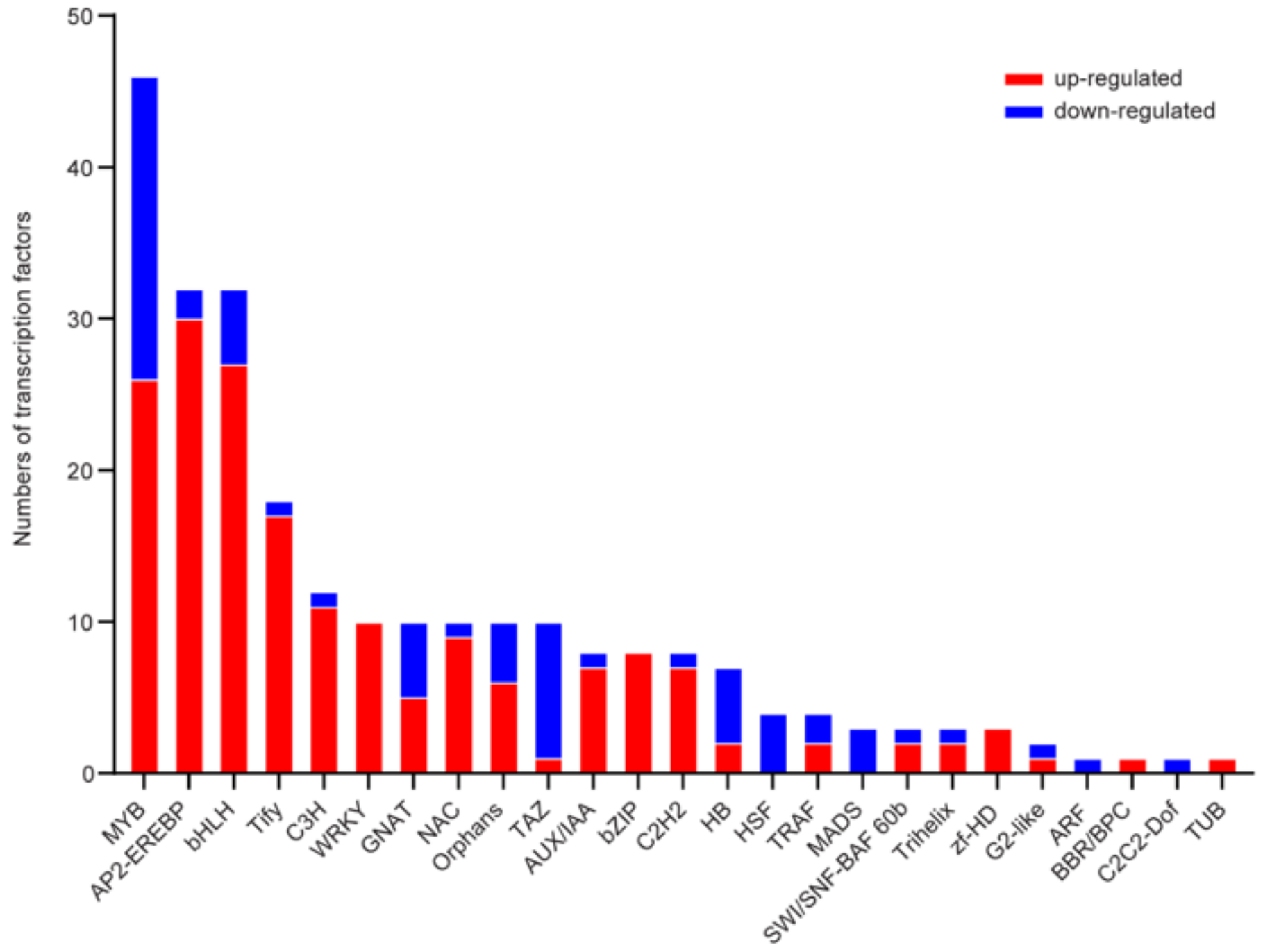

Figure 6

Numbers of trichome-related TFs among DEGs 


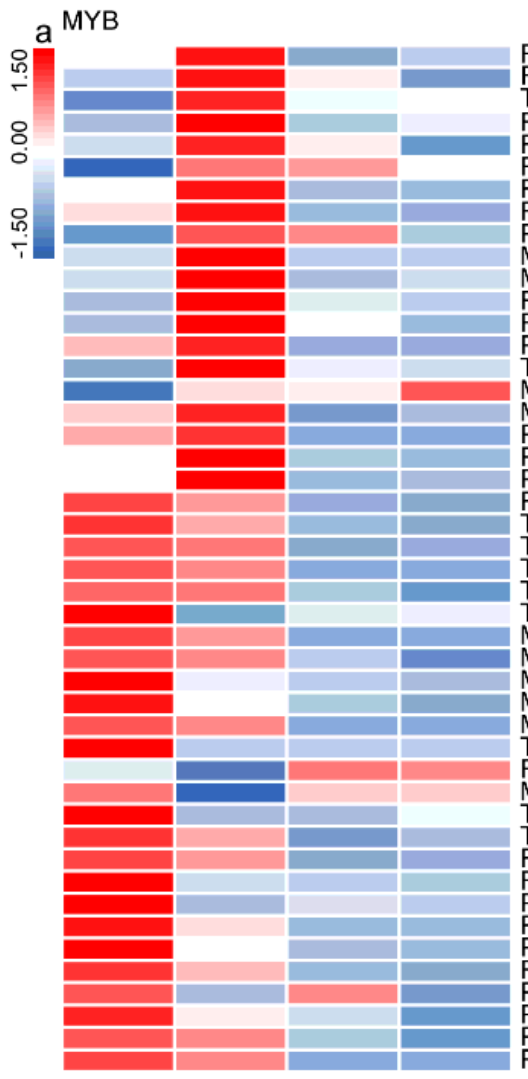

R2R3-MYB transcription factor - 1 Transcription factor MYB44-1 R2R3-MYB transcription factor -3 R2R3-MYB transcription factor -4 R2R3-MYB transcription factor -4 R2R3-MYB transcription factor -6 R2R3-MYB transcription factor -7 Protein REVEILLE

MYB transcription factor

MYB transcription factor

R2R3-MYB transcription factor - 8

R2R3-MYB transcription factor -8 R2R3-MYB transcriptic

ranscription factor MYB44-2

MYB-related protein 308

MYB transcription factor

Protein RADIALIS-2

Protein RADIALIS-3

2R3-MYB transcription factor-10

ranscription factor MYB44-3

Transcription factor MYB44-4

ranscription factor MYB44-5

ranscription factor MYB44-6

MYB-related protein MYB4-1

MYB-related protein MYB4-2

MYB-related protein MYB4-3

MYB-related protein MYB4-4

MYB-related protein MYB4-5

ranscription factor MYB44-8

R2R3-MYB transcription factor-11

MYB-related protein 308

ranscription factor MYB12

2 3 -MYB transcription factor-

R2R3-MY

Protein RADIALIS-5

Protein RADIALS-

Protein RADIALIS-7

R2R3-MYB transcription factor-14

Protein RADIALIS-8

Protein RADIALS- 9

Protein RADIALIS-11

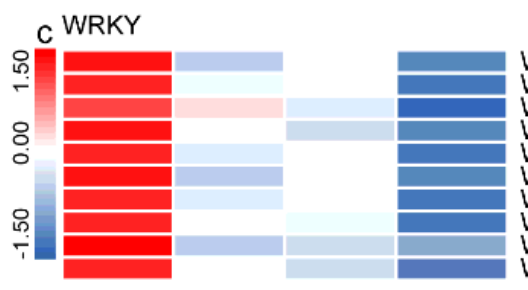

WRKY transcription factor 54 WRKY transcription factor 41-1 WRKY transcription factor 53 WRKY transcription factor 25 WRKY transcription factor 11 WRKY transcription factor 48 WRKY transcription factor 41-2 WRKY transcription factor 24 WRKY transcription factor 41-3 WRKY transcription factor 50

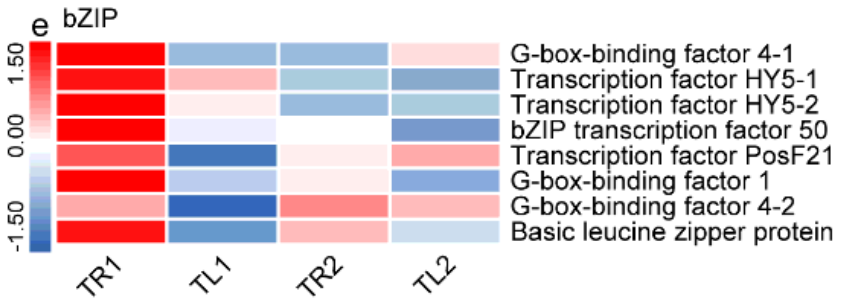

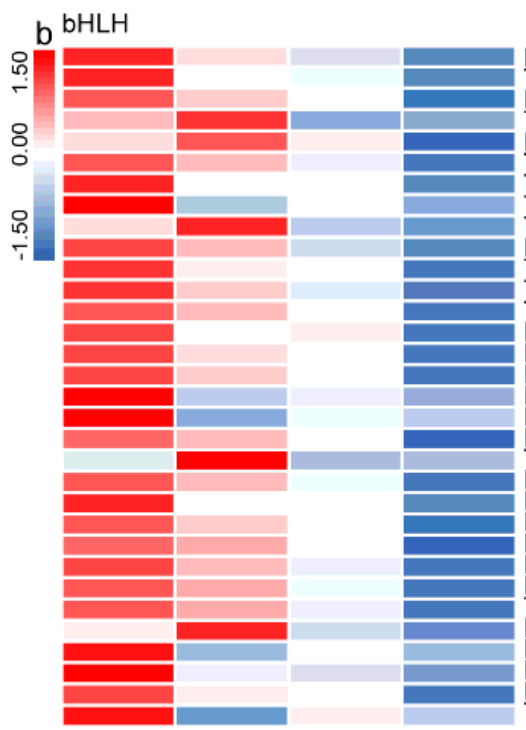

Hypothetical protein VITISV LH100-1 Protein A4U 43-1 (100-2 Protein A4U43-2

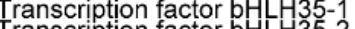

ranscription factor bH

Transcription factor ORG2 Hypothetical protein PRUPE Transcription factor bHLH148-2 Transcription factor bH년 $35-1$

Transcription factor bH 1 H $35-2$
Protein LOC $105047972-1$

Protein A4U 43-3

Protein A4U 43-4

rotein A4U43-5 Hypothetical pro Transcription factor bHLH100-3 rotein LOC105056299-1 Protein LOC $105056299-2$ Protein LOC105056299-3 Protein LOC 103705848 Protein LOC 105047972-3 Transcription factor bH Hypothetical protein MIMGU Protein LOC 103701976-1 Transcription factor bHLH49

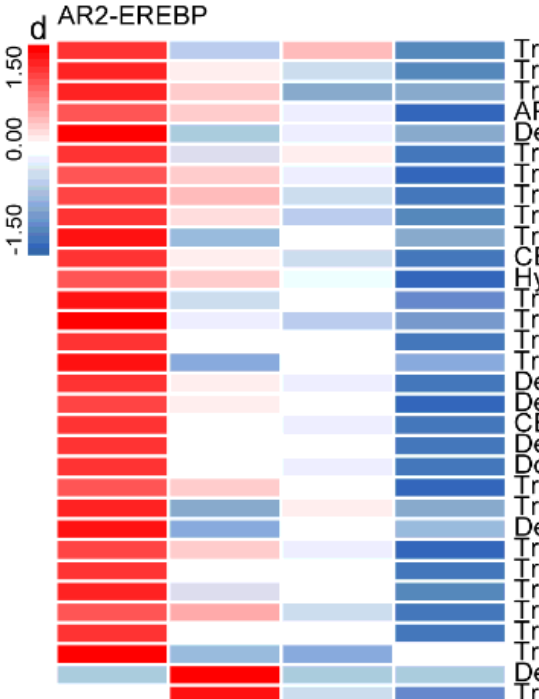

Transcription factor ERF109-1 Iranscription factor ERF060-1 AP 2 domain -containing protein Dehydration-responsive protein $1 \mathrm{C}-1$ ranscription factor ERF 27 ranscription factor 2 ranscription factor ERF109-2 (109-3 政 yypothetical protein POPTR ranscription factor ERFO 1 ranscription factor ERF061-2 ranscription factor $1 \mathrm{~A}-1$

ranscription factor 2-2

Dehydration-responsive protein $1 \mathrm{G}-1$

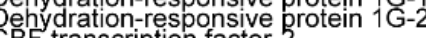
$\mathrm{CBF}$ transcription factor-2 - ehydration-responsive protein $1 \mathrm{C}-2$ ranscription factor ERF 11 ranscription factor 8-1 作-3 ranscription factor ERF109-4 ranscription factor ERF 109-5 ranscription factor $1 \mathrm{~A}-2$ ranscription factor 2-3 ranscription factor $8-2$ Tactor randration-responsive protein $1 \mathrm{~F}$

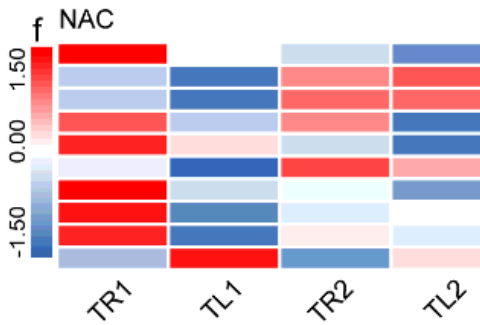

NAC domain-containing protein $68-1$ NAC domain-containing protein 83-1 NAC domain-containing protein 18 NAC domain-containing protein 41 NAC domain-containing protein 68-2 NAC domain-containing protein $21 / 22$ NAC domain-containing protein 83-2 NAC domain-containing protein 2 NAC transcription factor 47 NAC domain-containing protein 48 से से से से

\section{Figure 7}

Heat map diagrams showed the relative expression levels of TFs possibly associated with trichome. a MYB family members; b bHLH family members; c WRKY family members; d AR2-EREBP, Ethyleneresponsive family members; e basic region/leucine zipper (bZIP) family members; f NAM/ATAF/CUC family members. The color scale on the top from blue to red represents FPKM values from low to high. The Gene IDs involved in this figure were listed in Table S4-S9. 

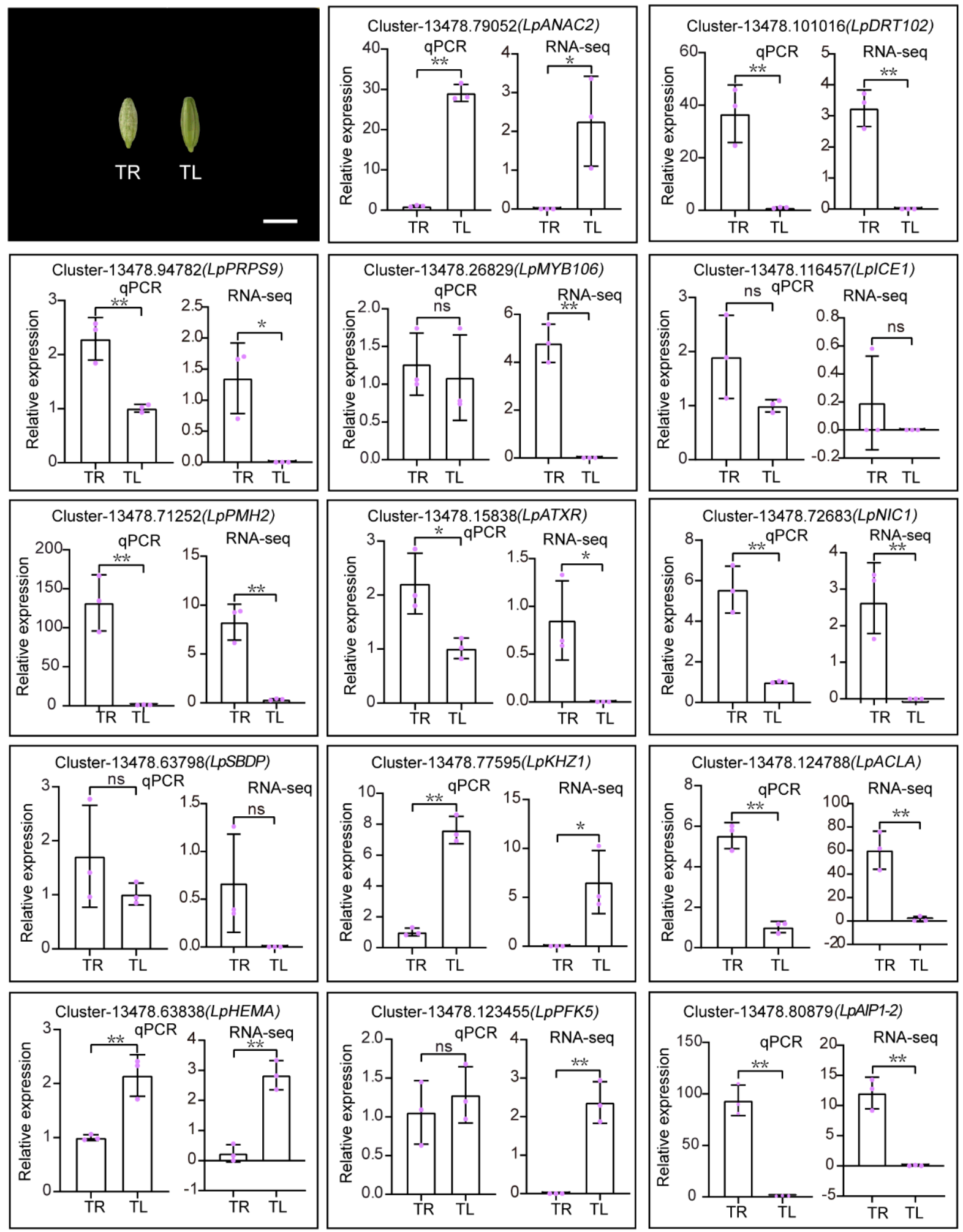

\section{Figure 8}

Expression analysis of the selected unigenes in the L. pumilum buds at $13.5 \mathrm{~mm}$ length stage. Scale bar $\bigotimes$ $10 \mathrm{~mm}$. The qRT-PCR values were examined by using the $2-\triangle \triangle \mathrm{CT}$ method. Error bars represent the standard deviation (SD) of three independent biological replicates. *: $P \leq 0.05$ and **: $P \leq 0.01$; ns: the difference was not significant. TL, trichomeless; TR, trichome. 

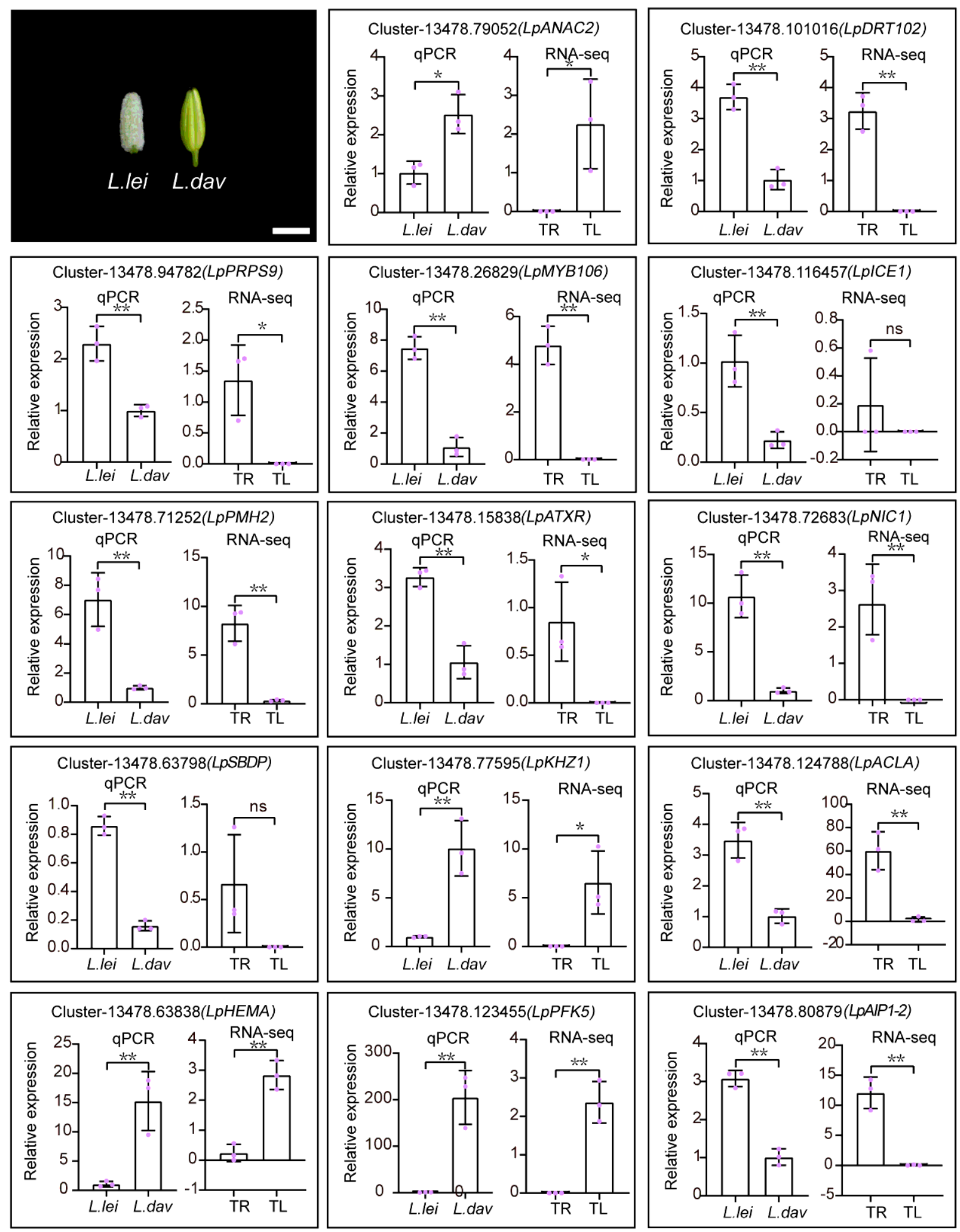

\section{Figure 9}

Expression analysis of the selected unigenes in the L. leichtlinii buds and L. davidii buds. All buds were $10 \mathrm{~mm}$ in length. Scale bar $₫ 10 \mathrm{~mm}$. The qRT-PCR values were examined by using the $2-\triangle \triangle C T$ method. Error bars showed the standard deviation (SD) of three independent biological replicates. *: $\mathrm{P} \leq 0.05$ and $\star *: P \leq 0.01$; ns: the difference was not significant. L. dav, Lilium. leichtlinii var. maximowiczii; L. lei, Lilium. davidii var. willmottiae; TL, trichomeless; TR, trichome. 


\section{Supplementary Files}

This is a list of supplementary files associated with this preprint. Click to download.

- PCRESupplementarymaterials.docx 